\title{
Modeling the Fluid Dynamics of Electrowetting on Dielectric (EWOD)
}

\author{
Shawn W. Walker and Benjamin Shapiro, Member, IEEE
}

\begin{abstract}
This paper discusses the modeling and simulation of a parallel-plate Electrowetting On Dielectric (EWOD) device that moves fluid droplets through surface tension effects. We model the fluid dynamics by using Hele-Shaw type equations with a focus on including the relevant boundary phenomena. Specifically, we show that contact angle saturation and hysteresis are needed to predict the correct shape and time scale of droplet motion. We demonstrate this by comparing our simulation to experimental data for a splitting droplet. Without these boundary effects, the simulation shows the droplet splitting into three pieces instead of two and the motion is over 15 times faster than the experiment. We then show how including the saturation characteristics of the device, and a simple model of contact angle hysteresis, allows the simulation to better predict the splitting experiment. The match is not perfect and suffers mainly because contact line pinning is not included. This is followed by a comparison between our simulation, whose parameters are now frozen, and a new experiment involving bulk droplet motion. Our numerical implementation uses the level set method, is fast, and is being used to design algorithms for the precise control of microdroplet motion, mixing, and splitting.

[1439]
\end{abstract}

Index Terms-Control, electrowetting, level set method, microfluidics, modeling, two-phase flow.

\section{INTRODUCTION}

W ELL-DESIGNED MEMS devices take advantage of the large surface to volume ratios found at the microscale. In particular, microfluidic devices often exploit surface tension forces to actuate or control liquids [1]-[3]. Electrowetting refers to using electrical fields to effectively modify surface tension effects [4]-[6] (see [7] and [8] for some fascinating experimental demonstrations).

Applications for these devices range from microfluid transport [9], mixing [10], dispensing [11], and "lab-on-a-chip" devices that automate functions like sensing and testing of biological samples [12], [13] to tunable optical fiber devices [14], [15] and reflective displays [16].

For this paper, we are concerned with modeling a specific variant of electrowetting called Electrowetting-On-Dielectric (EWOD) [17], which has an extra insulating layer to enhance its operation. See [18] for an initial experimental demonstration

Manuscript received September, 2004; revised November, 2005. This work was supported by DARPA under Grant FCP0.0205GDB191, contact monitor Dr. Anantha Krishnan. Subject Editor F. K. Forster.

S. W. Walker is with the Department of Aerospace Engineering, University of Maryland, College Park, MD 20742 USA (e-mail: swalker@wam.umd.edu).

B. Shapiro is with the Department of Aerospace Engineering and the Bio-Engineering Department, University of Maryland, College Park, MD 20742 USA (e-mail: benshap@eng.umd.edu).

Digital Object Identifier 10.1109/JMEMS.2006.878876 and [19] for an analysis of the advantage of using a dielectric insulating layer in an electrowetting system.

Similar applications exist for the EWOD device as well, such as mass spectrometry [20], mixing [21], "lab-on-a-chip" [22], micro-injection [23], and particle separation and concentration control [24]. The potential uses of these technologies could be for controlled mixing of chemicals and automated DNA testing.

Ultimately, these applications will need accurate fluid dynamical control in order to execute their many subtasks (i.e., particle control, precise droplet motion, splitting, optimal mixing, etc.). But this will also require accurate models to help design robust controllers as well as guide device optimization. Furthermore, these models must be convenient and cheap to use in order to fit within available control design and optimization methodologies.

Other modeling efforts of EWOD include [17], which gives a basic model of how the device parameters affect droplet splitting. Equilibrium models for the shape of sessile drops on a charging dielectric plate are given in [25] and [26]. In particular, [25] considers a conducting liquid on top of an insulating layer and the effect of charge trapping at high voltage on contact angle saturation. In [26], it is shown that liquid resistance can lead to contact angle saturation in the EWOD devices. An alternative, lumped parameter, electromechanical model for a one-dimensional (1-D) liquid column actuated by electrowetting is given in [27] for the equilibrium case and in [28] for the dynamics. In addition, a dynamic model of the contact angle variation for a spreading axisymmetric drop is given in [29]. And recently in [30], a diffuse interface model and simulation of droplet motion is compared to experiments on a scaled-up version of the electrowetting device.

In this paper, we present a distributed parameter model of EWOD fluid dynamics that is able to approximately capture the evolution of a droplet's liquid-gas interface, using the level set method [31], in two dimensions. Our model includes a rough approximation of contact angle hysteresis, which is different than, though analogous to, the contact line friction model discussed in [28] and [29]. Furthermore, the simulation of our model is sufficiently fast and low dimensional to use in controller design.

This paper is organized as follows. Section II gives an overview of the EWOD device's form and function. Section III develops the governing fluid equations and boundary conditions, along with our model of contact angle hysteresis. Section IV describes the numerical solution scheme, which uses a level set method for tracking the droplet boundary. And Section V presents our numerical results in comparison with two experiments that exhibit droplet splitting and bulk droplet motion. 


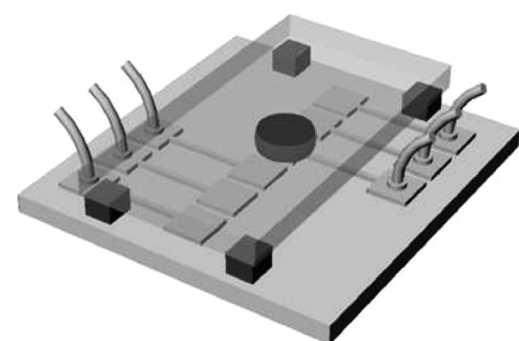

Fig. 1. Schematic of sample EWOD device (courtesy of C. J. Kim at UCLA).

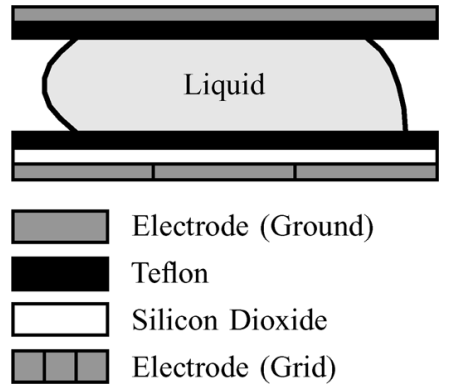

Fig. 2. Cross-sectional view of the EWOD device.

\section{DESCRIPTION OF THE DEVICE}

A schematic of an EWOD device is given in Fig. 1, while Fig. 2 shows a cross-sectional view. The device consists of a sandwich of various layers listed from top to bottom as: top (transparent) electrode, hydrophobic Teflon coating, droplets of water (here only one droplet is shown), another Teflon coating, a layer of solid dielectric silicon dioxide, and an underlying grid of electrodes. There are also spacers to ensure that the channel height is uniform.

The basic principle of operation is that the liquid-gas interface of the droplet can be locally deformed by capacitively charging the silicon dioxide layer underneath it. The induced motion of the droplet is due to competing effects of energy storage between the dielectric layer and the surface energy of the liquid-gas interface [26]. Effectively, each electrode can change the surface tension properties immediately above it. This change can be used to move droplets from electrode to electrode, to split droplets (by pulling on either side using three electrodes), to join droplets by making them collide, and to mix fluid in droplets by making the droplets execute complex paths.

An experimental device with a splitting droplet is shown in Fig. 3 (the view is through the top transparent electrode). The actuation voltages of the three electrodes from left to right are 25,0 , and $25 \mathrm{~V}$.

In [26], we presented a model for the equilibrium shape of droplets under applied electric fields. In this paper, we further consider the nonequilibrium fluid dynamics. Specifically, we focus on modeling and simulating motion, splitting, and joining of the liquid droplets.

\section{EWOD MODELING}

This section describes the EWOD modeling approach. In particular, our main assumptions, derivation of the fluid equations,

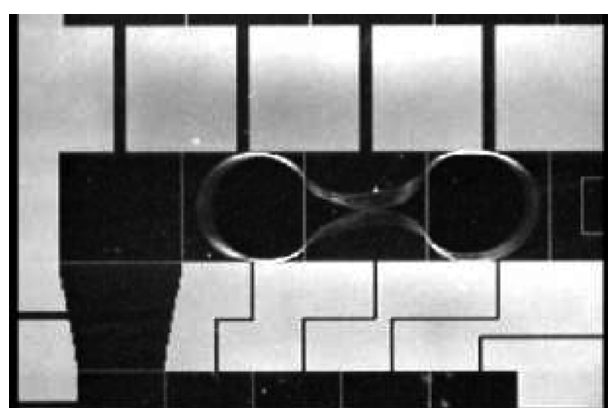

Fig. 3. Top view of experimental EWOD device (courtesy of C. J. Kim at UCLA).

TABLE I

PHYSICAL PARAMETERS

\begin{tabular}{c|c} 
Parameter & Symbol Definition \\
\hline Surface Tension & $\sigma_{l g}=0.07199 \mathrm{~J} / \mathrm{m}^{2}$ \\
\hline Dynamic Viscosity & $\mu=0.89 \mathrm{~g} / \mathrm{m} \cdot \mathrm{s}$ \\
\hline Density & $\rho=996.93 \mathrm{Kg} / \mathrm{m}^{3}$ \\
\hline Channel Height & $H=70 \mu \mathrm{m}$ \\
\hline Electrode Length & $L_{E l e c}=1.4 \mathrm{~mm}$ \\
\hline Length Scale & $L \approx 3 \times L_{E l e c}$ \\
\hline Velocity Scale & $U_{0}(\mathrm{see} \mathrm{Sec} . \mathrm{V}-\mathrm{A})$ \\
\hline Time Scale & $t_{0}=L / U_{0}$ \\
\hline Pressure Scale & $P_{0}=\sigma_{l g} / L$ \\
\hline Reynolds Number & $\mathrm{Re}=\rho U_{0} H / \mu$ \\
\hline Capillary Number & $\mathrm{Ca}=\mu U_{0} / \sigma_{l g}$ \\
\hline & \multicolumn{1}{|c|}{\begin{tabular}{c} 
Top View \\
\hline
\end{tabular}} \\
\hline
\end{tabular}

Fig. 4. EWOD device geometry. The coordinate axes are defined such that the top and bottom plates of the device lie in planes parallel to the $x-y$ plane. The physical parameters of the device are listed in Table I.

proper boundary conditions, voltage actuation, contact angle saturation, and hysteresis effects are discussed in detail. A list of the physical parameters for the geometry of the EWOD device, as well as the fluid parameters for distilled water at standard temperature and pressure (assumed in our model), is given in Table I.

\section{A. Governing Equations of the Liquid Flow}

In the following sections, the main assumptions and governing equations for the flow of liquid inside an EWOD device are described (see Fig. 4). In particular, we obtain a model similar to Hele-Shaw type flow with pressure boundary conditions at the liquid-gas interface proportional to its mean curvature.

1) Navier-Stokes Equations: We start by considering the Knudsen number of the EWOD device, which provides a measure of how accurate the continuum hypothesis is for a fluid 
system [32]. For our case, we can assume the flow physics to be a continuum because the Knudsen number is

$$
\mathrm{Kn}=\frac{\lambda_{\text {air }}}{H}=\frac{6.111 \times 10^{-8} \mathrm{~m}}{70 \times 10^{-6} \mathrm{~m}}=8.73 \times 10^{-4}
$$

where $\lambda_{\text {air }}$ is the mean free path of air molecules at standard temperature and pressure, and $H$ is the channel height of the device. Clearly, this is within the continuum regime which is defined to be $\mathrm{Kn}<10^{-2}$. The Knudsen number associated with the liquid flow is even smaller because the mean free path of water is much lower than that of air.

Since the flow is a continuum, the dimensional Navier-Stokes equations are applicable. Because we are modeling the flow of water, incompressibility and Newtonian fluid assumptions may be used [33]. This gives

$$
\begin{aligned}
\nabla \cdot \bar{V} & =0 \\
\rho\left(\bar{V}_{t}+\bar{V} \cdot \nabla \bar{V}\right) & =-\nabla P+\mu \nabla^{2} \bar{V}
\end{aligned}
$$

in the bulk liquid, where $\bar{V}=(u, v, w)$ is the three dimensional velocity, $P$ is the pressure, subscript $t$ denotes the partial derivative with respect to time, and $\rho$ and $\mu$ are the density and dynamic viscosity, respectively. Equations (1) and (2) represent conservation of mass and momentum, respectively, with gravity ignored because the potential energy change in the $z$ direction is negligible when the channel height, $H$, is small.

Next, we have the boundary conditions for a liquid droplet between two parallel plates. On the top and bottom plates, we have the usual no-slip condition for velocity (i.e., all velocity components are zero). Since the air surrounding the droplet is not being forced, it does not significantly affect any droplet motion. Therefore, by ignoring the airflow, we have the following conditions for the free surface of an incompressible, Newtonian liquid (i.e., the liquid-gas interface) [34]

$$
\begin{aligned}
\hat{n} \cdot \mathrm{T} \hat{n} & =-\sigma_{\lg } \kappa \\
\hat{t} \cdot \mathrm{T} \hat{n} & =0
\end{aligned}
$$

where $\sigma_{\lg }$ denotes surface tension, $\kappa$ is the mean curvature of the interface [35], $\mathrm{T}$ is the stress tensor, $\hat{n}$ is the unit normal vector to the interface, and $\hat{t}$ is any tangent vector to the interface. Physically, (3) states that the normal stress across the liquid-gas interface is balanced by surface tension, whereas (4) says the tangential stress vanishes because the airflow is negligible.

2) Hele-Shaw Type Flow: Since we have pressure-driven flow in a slot with channel height much smaller than the diameter of the droplet [33], the Reynolds number is small and we assume the flow can be modeled in two dimensions. By making the additional assumption that the $x$ and $y$ fluid velocity components $u$ and $v$ have a quadratic profile in the $z$ direction (i.e., local Poiseuille flow; see Fig. 5), (1) and (2) can be nondimensionalized and reduced to a form similar to Hele-Shaw flow [34]:

$$
\begin{aligned}
\nabla^{2} P & =0 \\
\left(\frac{L}{H} \operatorname{Re}\right) u_{t}+12\left(\frac{L}{H}\right)^{2} u & =-\frac{P_{0} L}{U_{0} \mu} P_{x}
\end{aligned}
$$

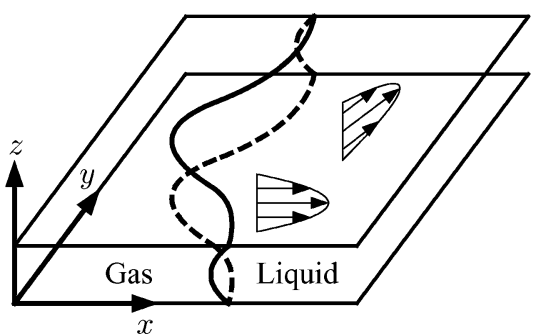

Fig. 5. Velocity profile: the fluid velocity field is assumed to have a quadratic profile in the $z$ direction.

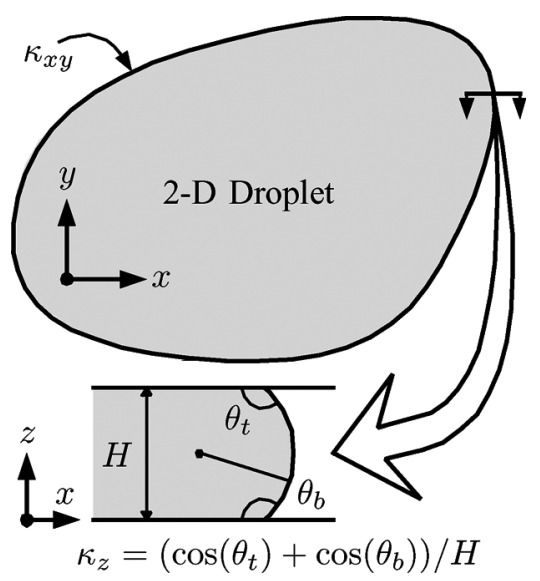

Fig. 6. Overhead view of a (2-D) droplet with side view zoom-in of the interface. The liquid-gas interface is assumed to have a circular cross-section, which gives an estimate of the $z$ curvature, $\kappa_{z}$, in dimensional form. The $x-y$ curvature, $\kappa_{x y}$, is just the curvature of the boundary of the 2-D droplet.

$$
\left(\frac{L}{H} \operatorname{Re}\right) v_{t}+12\left(\frac{L}{H}\right)^{2} v=-\frac{P_{0} L}{U_{0} \mu} P_{y}
$$

where the term on the far left of (6) and (7) is the extra term beyond the usual Hele-Shaw equations. This time derivative term is included because it may have a large magnitude due to rapidly varying pressure boundary conditions if high-frequency voltage actuation is used to modulate the droplet's contact angles.

The boundary conditions for (5) are then given by the Young-Laplace relation [34], which says (in nondimensional form) that the pressure on the liquid-gas interface is equal to the mean curvature of the interface. Because the channel spacing is so small, this is accurately approximated by

$$
P=\kappa_{x y}+\frac{L}{H} \kappa_{z}, \quad \text { at the liquid/gas interface }
$$

where $\kappa_{x y}$ is the nondimensional curvature of the droplet in the $x-y$ plane, $\kappa_{z}$ is the nondimensional curvature of a cross-section of the droplet along the $z$ axis (see Fig. 6), and $L$ is the $x-y$ length scale of the device. Given that (5) has been posed in two dimensions, (8) is evaluated at each point of the two-dimensional (2-D) droplet boundary and is discussed in Section III-B1.

\section{B. Physics of the Droplet Boundary}

Above, we described the governing equations of liquid droplet motion. We now discuss the geometry and different physical phenomena happening at the liquid-gas interface, such 


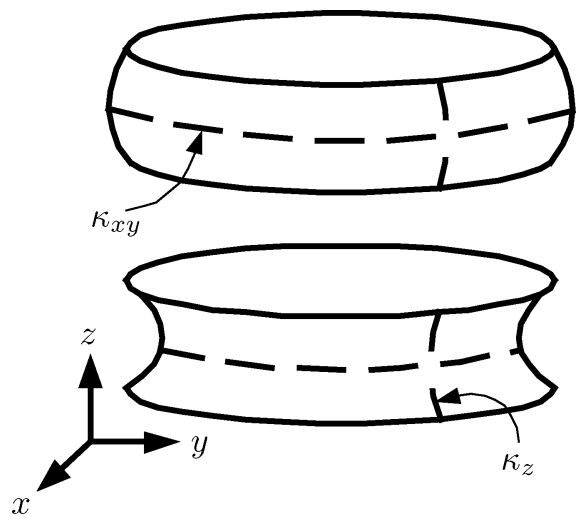

Fig. 7. Curvature note: $\kappa_{x y}$ and $\kappa_{z}$ are both positive for the bulging droplet on top. For the inward bending droplet, $\kappa_{z}$ is negative.

as voltage actuation, contact angle saturation, and hysteresis. We show how these effects are modeled and how they affect the computation of the boundary conditions.

1) Interface Curvature: The interface mean curvature is approximated using the individual curvatures $\kappa_{x y}$ and $\kappa_{z}$ in (8). We compute the $z$ curvature by assuming the interface has a circular cross-section (see Fig. 7). The $x-y$ curvature computation requires a representation of the shape of the two dimensional droplet boundary. This is accomplished by using a level set method to implicitly capture the interface and is described in more detail in Section IV-C2.

To use the circular approximation for computing the $z$ curvature, we must know the slope of the liquid-gas interface crosssection at the floor and ceiling of the EWOD device. This is given by the top and bottom contact angles, $\theta_{t}$ and $\theta_{b}$ respectively (see Fig. 6). After some basic geometry, the dimensional $z$ curvature is given by

$$
\kappa_{z}=-\frac{1}{H}\left[\cos \left(\theta_{t}\right)+\cos \left(\theta_{b}\right)\right]
$$

which gives the nondimensional curvature as

$$
\kappa_{z}=-\left[\cos \left(\theta_{t}\right)+\cos \left(\theta_{b}\right)\right]
$$

Through the use of EWOD, contact angles can be voltage controlled, thereby allowing the droplet pressure field to be actuated through the boundary condition (8). The details of voltage actuation and contact angles are described in the next Sections III-B2 and III-B3.

2) EWOD Charging Time: We analyze the electrical charging time of the EWOD device to determine if it must be included in our model. Consider the circuit diagram shown in Fig. 8. Using transfer function theory [36], which reduces the ordinary differential equation associated with the electric circuit to an algebraic problem, we can obtain an estimate for the time constant of the device. The transfer function for this circuit is given by

$$
T(s)=\frac{\alpha_{1} s+\alpha_{2}}{\alpha_{3} s^{2}+\alpha_{4} s+\alpha_{5}}
$$

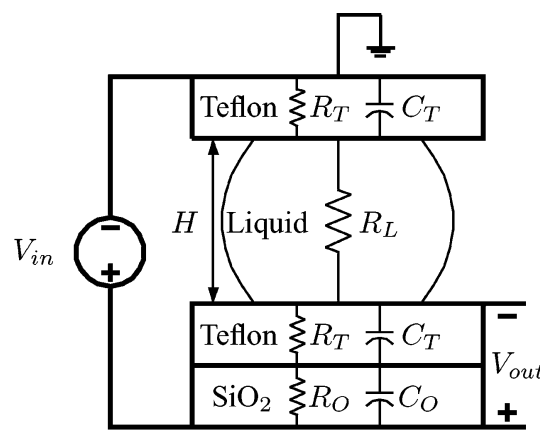

Fig. 8. EWOD-lumped circuit schematic.

where the coefficients are given by

$$
\begin{aligned}
& \alpha_{1}=\left(C_{T}+C_{O}\right) R_{O} R_{T} \\
& \alpha_{2}=R_{O}+R_{T} \\
& \alpha_{3}=R_{L} R_{O} C_{O} R_{T} C_{T} \\
& \alpha_{4}=R_{O} R_{T}\left(C_{T}+2 C_{O}\right)+R_{L}\left(R_{O} C_{O}+R_{T} C_{T}\right) \\
& \alpha_{5}=R_{O}+R_{L}+2 R_{T} .
\end{aligned}
$$

Using the parameters for water, silicon dioxide, Teflon, and the dimensions of the EWOD device [17], (10) becomes

$$
T(s)=\frac{1927.5\left(s+3.957 \times 10^{-6}\right)}{\left(s+3.132 \times 10^{-6}\right)(s+2439.7)} .
$$

After approximately canceling the two near-identical terms in the numerator and denominator, we are left with a transfer function describing a first-order differential equation. The defining parameter of any first order system is the time constant, which in this case is $0.41 \mathrm{~ms}$. Using this, the electrical charging time is estimated as four times the time constant, or $1.64 \mathrm{~ms}$.

For the splitting droplet experiment in Section V-A, the time to split is $0.167 \mathrm{~s}$. Since the majority of the voltage drop occurs across the bottom $\mathrm{SiO}_{2}$ and Teflon layer, and the charging time is over 100 times faster than the bulk fluid motion we are interested in (i.e., droplet splitting), we assume the output voltage is instantaneously equal to the input voltage. Therefore, given that there is a direct relation between contact angle and applied voltage (see Section III-B3), EWOD is capable of changing the contact angle very quickly.

3) Contact Angles and Saturation: There is a considerable amount of literature on contact angles and wetting phenomena; see the following references for a sampling [37]-[40]. For a detailed physical description of electrowetting, see [41]-[44], [25]. In this section, we are concerned with how the contact angle varies with respect to the applied voltage.

According to [4], [45], and [29], for a sessile drop on a single dielectric plate, the Young-Lippmann equation predicts a near parabolic curve relating contact angle to the capacitive voltage across the plate (see Fig. 9). However, if Young-Lippmann is used to simulate droplet splitting, it predicts an incorrect shape for the motion of the droplet. This is because electrowetting, in reality, deviates from the Young-Lippmann theory at high voltages and reaches a saturation limit (also shown in Fig. 9). 


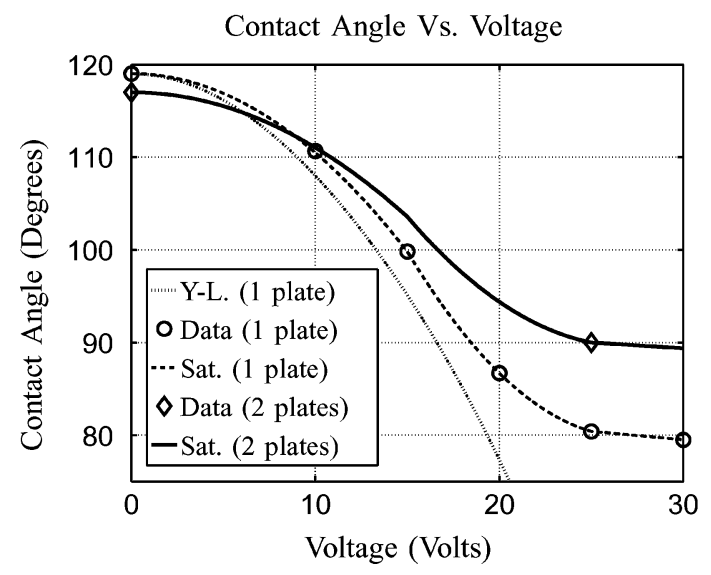

Fig. 9. Contact angle versus voltage curves. Theoretical and experimental data for contact angle variations using electrowetting on dielectric are presented. The dotted line denoting the Young-Lippmann (Y-L) curve is theoretical [4], [45], [29]. The single plate saturation curve has six experimental data points (given in [17]) with a piecewise interpolating polynomial (see dashed line and "o" data points). The two plate saturation curve has two experimental data points (also from [17]) with an interpolating curve derived from the single plate case in Section III using a linear map (see solid line and " $\diamond$ " data points). This curve is used in our simulation to predict the correct droplet motion and splitting time (see Section V).

In Sections V-A1 and V-A2, we present simulations using the Young-Lippmann equation and saturation, respectively, to illustrate the importance of modeling the latter. For more information on the causes of contact angle saturation of sessile droplets, see [26].

The available literature only discusses a sessile droplet on a single plate. For this paper, we need data on contact angle variations of a droplet sandwiched between two plates. In [17], experimental contact angle data for the EWOD device is given at an applied voltage of 0 and $25 \mathrm{~V}$. The top contact angle remains approximately the same at $117^{\circ}$ regardless of voltage actuation. This is because most of the dielectric energy is stored in the bottom plate due to the presence of the $\mathrm{SiO}_{2}$ layer. Therefore, we assume the nominal contact angle on the top plate is fixed at $117^{\circ}$. The bottom contact angle varies between $117^{\circ}$ and $90^{\circ}$ at 0 and $25 \mathrm{~V}$, respectively.

In order to model contact angle variations on the bottom plate for any voltage, we must combine the two experimental data points for the parallel plate case with the six data points for the single plate case (shown in Fig. 9). Since there is an overlap between two of the data points for both cases, we define a linear mapping that represents this correspondence

$$
\theta_{2}=\frac{117^{\circ}-90^{\circ}}{119^{\circ}-80.4^{\circ}}\left(\theta_{1}-80.4^{\circ}\right)+90^{\circ}
$$

where $\theta_{1}$ is the contact angle for a single plate and $\theta_{2}$ is the contact angle for two plates. This equation maps $119.0^{\circ}$ to $117^{\circ}$ and $80.4^{\circ}$ to $90^{\circ}$. By taking the six data points, and their piecewise interpolating polynomial, for saturation on a single plate as input to this linear map, we obtain the saturation curve for two plates as output (see Fig. 9). Due to the scarcity of data on

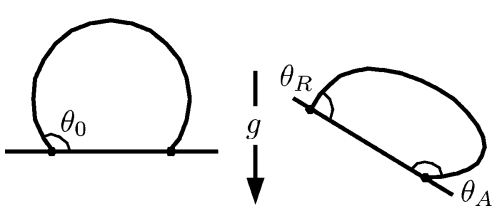

Fig. 10. Illustration of contact line pinning and hysteresis. The contact line of the droplet is where the liquid-gas interface meets the solid surface. Line pinning simply means the contact line (and the droplet) is stuck to the surface. A direct result of this is contact angle hysteresis, which refers to the situation where the receding angle $\theta_{R}$ is less than the nominal (equilibrium) angle $\theta_{0}$, while the advancing angle $\theta_{A}$ is greater than $\theta_{0}$. In the diagram above, $\theta_{0}$ is the contact angle of the droplet on a horizontal surface, whereas $\theta_{R}$ and $\theta_{A}$ are the contact angles when the surface is tilted. The droplet can slide by using a large enough tilt angle, but the motion will be limited by the static frictional effect of line pinning and contact angle hysteresis will still be present. A similar situation happens in EWOD, where hysteresis also acts as a retarding effect by deforming the liquid-gas interface shape in an unfavorable way (see Section III-B4).

contact angle variation for the parallel plate EWOD device, we assume the two plate saturation curve in Fig. 9 is true for our model.

4) Hysteresis: Contact angle hysteresis is the last piece of physics we need to complete our model of droplet motion using EWOD forces. Hysteresis refers to the difference in contact angles between the advancing and receding ends of sessile drops. It is a direct consequence of contact line pinning, which acts as a force that resists any sliding motion, and can be seen when water droplets stick to the side of a solid surface (see Fig. 10). For more information on contact angle hysteresis and line pinning, see [37], [39], and [40].

From Fig. 10, for a sessile drop on a single plate, it can be seen that the advancing and receding contact angles are greater and smaller, respectively, than the nominal contact angle. This is also true for a droplet inside the EWOD device (shown in Fig. 11). Ideally, if there were no hysteresis, the nominal contact angle at the interface of the droplet should be determined by the two plate saturation curve in Fig. 9 and the applied voltage at the interface. But in the presence of hysteresis, the contact angles deflect from their nominal values which affects the pressure on the boundary by the Young-Laplace relation (8).

To see how it is affected, consider a circular droplet in motion due to voltage actuation (see Fig. 11). Let $\bar{P}_{A}$ and $\bar{P}_{R}$ denote the pressures at the advancing and receding ends of the droplet, respectively, when no hysteresis is present. And let $\widetilde{P}_{A}$ and $\widetilde{P}_{R}$ denote the same pressures with hysteresis. It is clear from Fig. 11 that the $z$ curvatures at the receding and advancing ends of the droplet are larger and smaller, respectively, for no hysteresis than with hysteresis. From (8), it can be seen that the curvature change implies that $\bar{P}_{R}>\widetilde{P}_{R}$ and $\bar{P}_{A}<\widetilde{P}_{A}$.

This change in boundary pressure weakens the pressure gradient throughout the droplet from what it would be without hysteresis because its magnitude is proportional to the pressure difference

$$
\begin{aligned}
& |\nabla \bar{P}| \propto\left|\bar{P}_{R}-\bar{P}_{A}\right| \\
& |\nabla \widetilde{P}| \propto\left|\widetilde{P}_{R}-\widetilde{P}_{A}\right|
\end{aligned}
$$




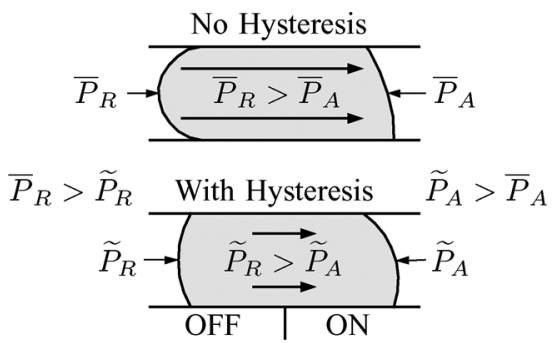

Fig. 11. Effects of contact angle hysteresis in the EWOD device. A droplet is shown moving from left to right due to voltage actuation (OFF/ON). When hysteresis is present, the contact angles differ from their nominal (nonhysteresis) values. The effects on the liquid-gas interface pressure are also shown. The presence of hysteresis causes the pressure gradient throughout the droplet to be weakened from the nominal case $\left(\bar{P}_{R}-\bar{P}_{A}>\widetilde{P}_{R}-\widetilde{P}_{A}\right)$.

where $\nabla \widetilde{P}$ and $\nabla \bar{P}$ are the pressure gradients with and without hysteresis, respectively. Using the fact that $\bar{P}_{R}>\widetilde{P}_{R}>\widetilde{P}_{A}>$ $\bar{P}_{A}$, we obtain the inequality

$$
|\nabla \widetilde{P}|<|\nabla \bar{P}|
$$

Hence, the driving force of the droplet motion is decreased when hysteresis is present. This is why our simulation (with just saturation modeled) predicts a split time over nine times faster than the experiment shows (see Section V.A).

From the discussion above, an obvious way to model hysteresis is to modify the contact angle of the interface based on which way it is moving. However, from our own numerical experiments, this method is not very robust. Therefore, we opted for a simpler model by assuming that

$$
\nabla \tilde{P}=K_{\text {hys }} \nabla \bar{P}
$$

where $K_{\text {hys }}$ is a constant smaller than one. In other words, we scale down the pressure gradient in (6) and (7) to account for hysteresis. This is analogous to the contact line friction model in [29] and [28], which also acts as a retarding effect on liquid motion.

Scaling the pressure gradient is simple and straightforward, yet is capable of approximately capturing the droplet motion and time scale observed in the experiments (see Section V.A.3). However, we do stress that it is not exact. It does not capture the effect of line pinning, which is observable in EWOD as demonstrated in [17] by the fact that droplets do not move unless sufficient voltage actuation is used. Line pinning is not completely understood and implementing a highly accurate model would be computationally expensive. Hence, we opted for a model that is computationally quick but still captures the lossy effect of droplet motion that is induced by line pinning; namely hysteresis.

We now estimate the hysteresis constant in terms of contact angles. From the relations given above, we have where the pressure terms are given by (8) and (9). Using these, $K_{\text {hys }}$ can be estimated by

$$
\begin{aligned}
& G_{\text {num }}= {\left[\cos \left(\theta_{t, 0 V}-\Delta_{\text {hys }}\right)+\cos \left(\theta_{b, 0 V}-\Delta_{\text {hys }}\right)\right.} \\
&\left.-\cos \left(\theta_{t, 25 V}+\Delta_{\text {hys }}\right)-\cos \left(\theta_{b, 25 V}+\Delta_{\text {hys }}\right)\right] \\
& G_{\text {den }}= {\left[\cos \left(\theta_{t, 0 V}\right)+\cos \left(\theta_{b, 0 V}\right)\right.} \\
& K_{\text {hys }}=\left.-\cos \left(\theta_{t, 25 V}\right)-\cos \left(\theta_{b, 25 V}\right)\right] \\
& G_{\text {num }}
\end{aligned}
$$

where $\Delta_{\text {hys }}$ is the extra amount of contact angle deflection from the nominal angle due to hysteresis, and $\theta_{t}$ and $\theta_{b}$ are the top and bottom contact angles, respectively. The voltage subscripts specify the actuation strength of the contact angles, with the top angle fixed at $117^{\circ}$ regardless of voltage and the bottom angle obeys the two plate saturation curve in Fig. 9. Due to the lack of data on hysteresis of EWOD driven droplets, we assume the contact angle deflection to be the same on the top and bottom of the advancing and receding ends of the droplet. In Section V.A.3, we use (12) to estimate the hysteresis angle deflection that corresponds to the appropriate constant, $K_{\text {hys }}$, that ensures the simulated splitting time matches the experiment.

\section{Final Equation Summary}

We now write the final model equations describing the fluid flow of a liquid droplet inside an EWOD device. The equations for the pressure field are

$$
\begin{aligned}
\nabla^{2} P & =0, & & \text { in } \Omega \\
P & =\kappa_{x y}+\frac{L}{H} \kappa_{z}, & & \text { on } \partial \Omega
\end{aligned}
$$

where $\nabla^{2}$ is the Laplacian operator, $\Omega$ denotes the domain of the liquid droplet in two dimensions, $\partial \Omega$ is its boundary, $P$ is the pressure, $L$ is a chosen length scale, $H$ is the channel height, and $\kappa_{x y}$ is the curvature in the $x-y$ plane. The $z$ curvature, $\kappa_{z}$, is given by

$$
\kappa_{z}=-\left[\cos \left(\theta_{t}\right)+\cos \left(\theta_{b}\right)\right]
$$

where $\theta_{t}$ and $\theta_{b}$ are the contact angles on the top and bottom of the EWOD device, respectively. The top angle is assumed to be 117 degrees regardless of the applied voltage. The variations of the bottom angle are given by the two plate saturation curve in Fig. 9.

The equations for the velocity field are

$$
\begin{aligned}
& \tau u_{t}+\beta u=-\frac{K_{\text {hys }}}{\mathrm{Ca}} P_{x}, \quad \text { in } \Omega \\
& \tau v_{t}+\beta v=-\frac{K_{\mathrm{hys}}}{\mathrm{Ca}} P_{y}, \quad \text { in } \Omega
\end{aligned}
$$

where $u$ and $v$ are the velocity components in the $x$ and $y$ directions, $K_{\text {hys }}$ is the hysteresis constant, and $C a$ is the capillary number. The constants $\tau$ and $\beta$ are given by

$$
K_{\mathrm{hys}}=\frac{\left|\tilde{P}_{R}-\tilde{P}_{A}\right|}{\left|\bar{P}_{R}-\bar{P}_{A}\right|}
$$

$$
\tau=\frac{\rho U_{0} L}{\mu} \quad \beta=12\left(\frac{L}{H}\right)^{2}
$$


where $U_{0}$ is a chosen velocity scale, $\rho$ is the fluid density, and $\mu$ is the dynamic viscosity.

Because we have two-phase flow, we need the following equation to describe the time-varying nature of the droplet domain

$$
\dot{\partial} \Omega=\left.\hat{n} \cdot(u, v)\right|_{\partial \Omega}
$$

where $\hat{n}$ is the unit normal vector of the boundary. Basically, the droplet boundary moves with the normal velocity of the fluid. In the next section, we discuss the numerical simulation of these equations.

\section{NUMERICAL IMPLEMENTATION}

The most crucial part of simulating the fluid equations in Section III-C is in handling the time-varying two-phase droplet boundary modeled by (19). Various methods for simulating two-phase flow are available (see [46] for a survey). For this paper, the method needs to be capable of simulating splitting and joining of droplets without excessive computation. Therefore, we chose the level set method (see [31], [47], [48]), which implicitly tracks the droplet boundary as the zero level set of a scalar function defined over the $x-y$ plane (see Fig. 14). This scalar function deforms and changes shape based on the fluid velocity field, which causes the zero level set to also change. Hence, the motion of the droplet boundary is captured through the evolution of the scalar function.

This level set function is used to define the domain of the droplet at each instant of time, allowing the pressure and velocity fields to be computed from finite difference approximations to (13), (14), (15), (16), (17). We combine these methods in a third order Runge-Kutta time-stepping algorithm which specifies an order to the computation of the pressure field, velocity field, and level set update (see Fig. 12). The following sections give the details of our algorithm which is based on the methods used in [49] and [48].

All simulations were performed with MATLAB on a Pentium $4,3.6 \mathrm{GHz}$ with $2 \mathrm{~GB}$ of RAM running Windows XP. The computing time of each simulation varied between three and six minutes for a $108 \times 108$ mesh, which shows the speed of our method.

\section{A. Discretization}

The computational domain is defined to be the unit square, and is discretized by a regular cartesian grid (see Fig. 13). For the simulations given in Section V, we used a $108 \times 108$ mesh. On this grid, the level set function, $\phi$, and the fluid variables $u, v$, and $P$ are sampled. A small buffer region, three grid nodes thick, is defined at the sides of the computational domain. No droplet motion is allowed inside the buffer region because of potential problems with computing second order spatial derivatives there.

\section{B. Initialization}

The level set function, $\phi$, is initialized to a signed distance function with the zero level contour corresponding to the initial interface shape (see Fig. 14). By distance function, we mean

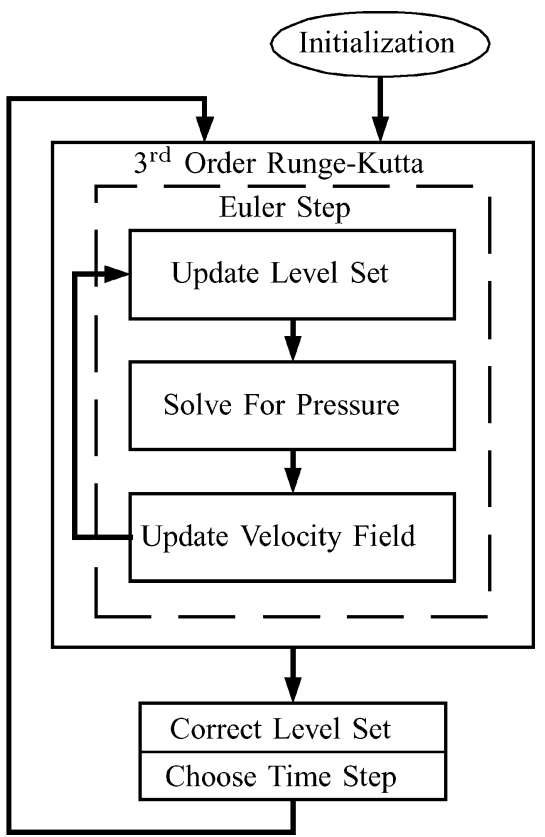

Fig. 12. Algorithm flowchart.

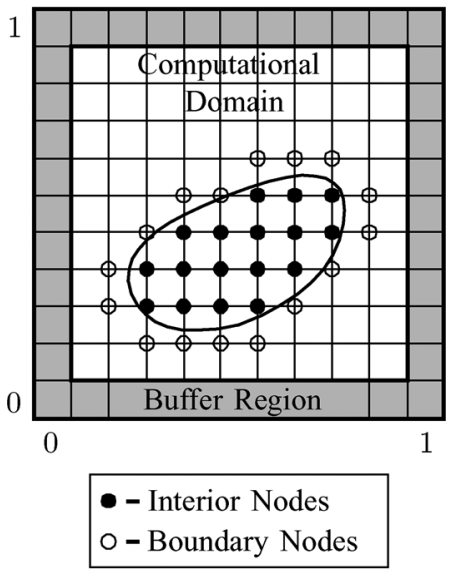

Fig. 13. Computational domain layout (liquid region corresponds to interior nodes).

that the value of $\phi$ at a grid point in the computational domain corresponds to the shortest distance that the grid point is from the interface. Signed distance means that $\phi$ is positive inside the droplet and negative outside. Next, the velocity field, $(u, v)$, is set to zero. And finally, we choose a small initial time step before entering the main update routine discussed in Section IV-C.

\section{Main Update Routine}

At each time step of our simulation, the fluid variables and level set function are updated by computing a convex combination of three forward Euler steps. This method is a third order Runge-Kutta method, and is detailed in [31] and [50].

In each Euler step, the level set is updated first, followed by the pressure, and then velocity. The updated level set is used in computing the pressure field for the new time step, which is then used to update the velocity field (see Fig. 12). In the following sections, we give the details of each of these subroutines. 


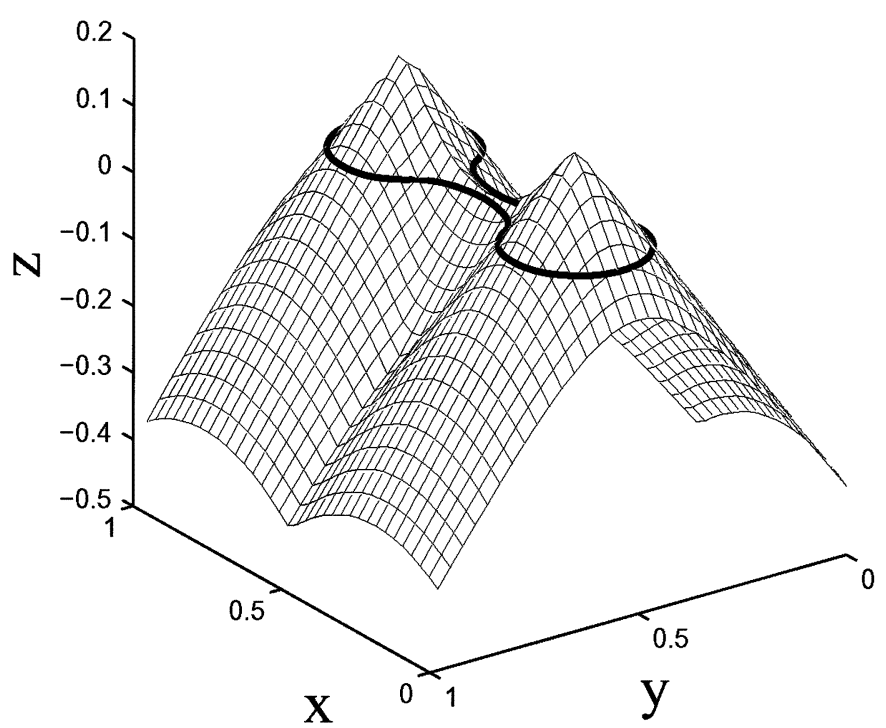

Fig. 14. Example of a level set function. The zero level contour is highlighted with a thick black line and shows a droplet about to split.

1) Update Level Set: The level set function is updated through a standard convection equation:

$$
\phi_{t}+(u, v) \cdot \nabla \phi=0
$$

This equation represents conservation of the quantity, $\phi$, while being transported by the velocity field, $(u, v)$. Hence, the zero level set "quantity," $\phi=0$, is transported by the local fluid velocity around the droplet boundary. The numerical implementation uses an upwinded third order Hamilton-Jacobi weighted essentially nonoscillatory (WENO) method for discretizing the velocity-gradient term, which uses $u, v$, and $\phi$ from the previous time step. This method is robust and is described in detail in [31].

In this paper, to reduce simulation time, only the grid nodes in a small band surrounding the zero level set are actually updated. This does not reduce accuracy since the whole level set must be reset to a distance function periodically (see Section IV-D1).

2) Solve for Pressure: Next, the domain, $\Omega$, of the droplet is defined to be the regions in the $x-y$ plane where the level set function, $\phi$, is positive (see Fig. 14). The computational domain of a hypothetical droplet is depicted in Fig. 13. Each of the grid nodes is located on an electrode with a known applied voltage. The local $x-y$ curvature of the boundary, $\partial \Omega$, is then given by [31]:

$$
\kappa_{x y}=-\left.\frac{\phi_{x}^{2} \phi_{y y}-2 \phi_{x} \phi_{y} \phi_{x y}+\phi_{y}^{2} \phi_{x x}}{\left(\phi_{x}^{2}+\phi_{y}^{2}\right)^{3 / 2}}\right|_{\phi=0}
$$

where the level set derivative terms are approximated using central differences. Because of fundamental problems with differentiating numerical data, the level set function, $\phi$, must be filtered prior to computing the derivative terms [50]. In addition, $\kappa_{x y}$ must be post-filtered to ensure smooth curvature data. This is mainly due to the explicit nature of the curvature calculation used here.

Then, we get the bottom contact angle, $\theta_{b}$, at each boundary node using the known voltage there and the two plate saturation curve in Fig. 9. Voltage transitions near the edge between two electrodes are smoothed out using linear interpolation, in a narrow region, to prevent large localized velocities caused by discontinuous boundary conditions. Finally, the boundary pressure values are computed using (14), (15), and (21) evaluated on the boundary nodes.

The pressure values at the interior nodes are computed by solving (13), which implicitly contains the conservation of mass (1). The numerical solution is obtained by using a simple GaussSeidel iterative solver with a relative error tolerance of $10^{-8}$ [51]. Other, more advanced methods for solving a matrix system of equations exist, but would require the matrix structure to be recreated at every time step since the domain of the droplet is always changing. In addition, the Gauss-Seidel solver is implemented in C, for speed, and called from MATLAB. Therefore, we saw no significant advantage with using a different method

Once the pressure values are known, the pressure gradient, $\left(P_{x}, P_{y}\right)$, at every interior node is computed using a central difference formula [51]. These values are then used in the velocity update routine.

3) Update Velocity Field: The fluid velocity components, $(u, v)$, obey two first order time differential equations given by (16) and (17). The pressure gradient provides a forcing term in the equations, which causes a velocity field to develop. We compute the velocities on our computational domain by discretizing (16) and (17) in space while keeping time continuous. This approach is commonly known as a semi-discrete method [52] (or method-of-lines) and allows for the use of an analytic solution to (16) and (17) for updating the velocity field.

For a time-invariant pressure gradient, the steady-state solutions of (16) and (17) are given by

$$
u_{s s}=-\frac{K_{\text {hys }}}{\mathrm{Ca} \beta} P_{x} \quad v_{\mathrm{ss}}=-\frac{K_{\mathrm{hys}}}{\mathrm{Ca} \beta} P_{y}
$$

Let $u_{i}$ and $v_{i}$ be the initial velocity components at time $t_{i}$. Then, by assuming the pressure gradient remains constant during the time step, $\Delta t$, the velocity field at $t_{i}+\Delta t$ is given by

$$
\left.(u, v)\right|_{t_{i}+\Delta t}=e^{-\Delta t \frac{\beta}{\tau}}\left(u_{i}, v_{i}\right)+\left[1-e^{-\Delta t \frac{\beta}{\tau}}\right]\left(u_{\mathrm{ss}}, v_{\mathrm{ss}}\right)
$$

where the above equation is the analytic solution to (16) and (17) evaluated at $t_{i}+\Delta t$. We apply this update to all interior grid nodes to obtain the velocity field inside the droplet for the current time step.

The last piece needed for updating the velocity is to extend it from inside the droplet to outside. In Section IV-C1, the velocity field is needed to update the level set function. But in order to do this properly, it must be extended into the boundary and exterior nodes of the computational domain. This is accomplished by letting the velocity components diffuse into the exterior region (see Fig. 15), which ensures a continuous velocity field for 


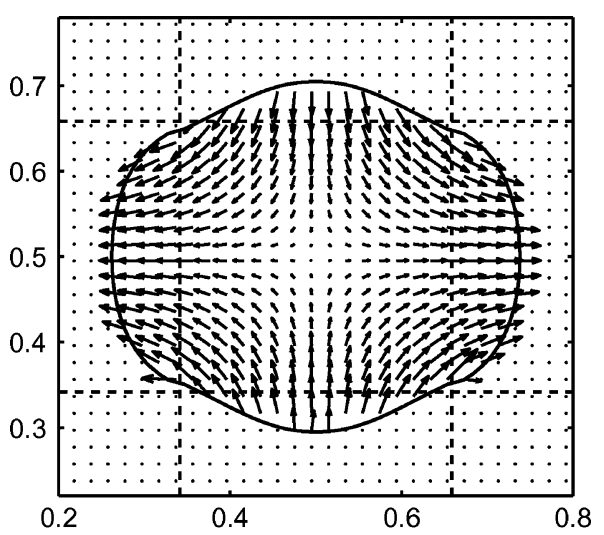

(a)

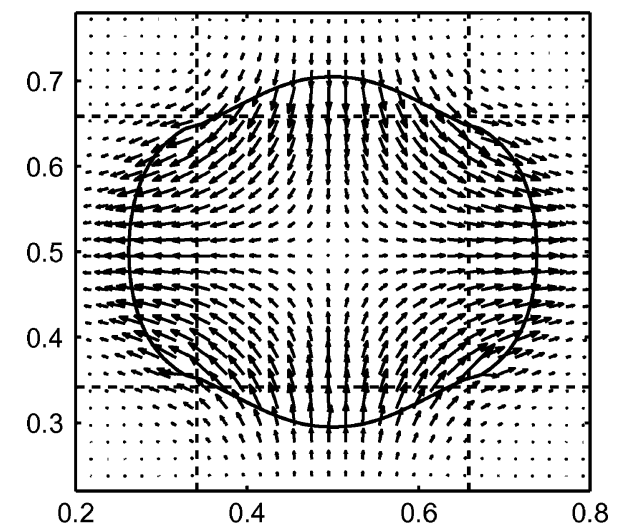

(b)

Fig. 15. Velocity field extension. Illustration showing before and after results of extending the velocity field: (a) Without extension and (b) with extension.

updating the level set. We now give the details of this extension algorithm.

First, fix the values of $u_{\mathrm{ss}}$ and $v_{\mathrm{ss}}$ at the interior grid nodes and set the edge node values of the computational domain to be zero. At each boundary and exterior grid node (excluding the edge nodes), compute a value for $u_{\mathrm{ss}}$ and $v_{\mathrm{ss}}$ using this formula:

$$
\left.u_{\mathrm{ss}}\right|_{i, j}=\frac{\left.u_{\mathrm{ss}}\right|_{i+1, j}+\left.u_{\mathrm{ss}}\right|_{i-1, j}+\left.u_{\mathrm{ss}}\right|_{i, j+1}+\left.u_{\mathrm{ss}}\right|_{i, j-1}}{4}
$$

where a similar formula is used for $v_{\mathrm{ss}}$ and $(i, j)$ are the row and column coordinates for each grid node. Iterate this process a fixed number of times for all boundary and exterior grid nodes. With each iteration, the velocity values diffuse further away from the interior region. For our simulations, we used 50 iterations to extend $u_{\mathrm{ss}}$ and $v_{\mathrm{ss}}$, which are then used in (22). This guarantees that the velocity field will always be extended. Our method is nothing more than Jacobi iterations executing on all boundary and exterior nodes and acting on velocity values. In [31] and [48], the method they use for extending the velocity field is based on a convection-type equation that propagates velocity data from the interior region into the boundary and exterior nodes. However, we do not use their method because it is computationally more complex. Another technique for generating velocity fields that also preserves the distance function property of the level set function is given in [47], but this is a
TABLE II

PinCH TIME VERSUS GRID RESOLUTION

\begin{tabular}{c|c|c} 
Grid Resolution & Pinch Time (ms) & $\%$ Dev. \\
\hline $84 \times 84$ & 127.9 & -0.9 \\
\hline $90 \times 90$ & 126.4 & -2.0 \\
\hline $96 \times 96$ & 125.5 & -2.7 \\
\hline $102 \times 102$ & 126.0 & -2.3 \\
\hline $108 \times 108$ & 129.0 & +0.0 \\
\hline $114 \times 114$ & 132.7 & +2.9 \\
\hline $120 \times 120$ & 133.9 & +3.8 \\
\hline $130 \times 130$ & 140.0 & +8.5 \\
\hline $140 \times 140$ & 131.2 & +1.7 \\
\hline $150 \times 150$ & 139.8 & +8.4 \\
\hline $161 \times 161$ & 125.0 & -3.1 \\
\hline $174 \times 174$ & 134.2 & +4.0 \\
\hline $187 \times 187$ & 125.4 & -2.8 \\
\hline $201 \times 201$ & 129.8 & +0.6 \\
\hline & &
\end{tabular}

very expensive computation. We prefer our method because it is simpler and gives excellent performance.

\section{Final Cleanup}

After updating the level set function, pressure, and velocity fields, there remain two final tasks. Reconditioning the level set function and choosing the next time step. Once completed, the program loops back to Section IV.C to continue the simulation.

1) Correct Level Set: Despite its ingenuity, the level set method does have problems. Since we are using the fluid velocity to update $\phi$, it is highly likely that the level set will become distorted and introduce numerical inaccuracies [47]. This requires periodically resetting $\phi$ so that it is always close to being a distance function. This is done by explicitly finding the zero level set of $\phi$, which represents the droplet boundary, and recomputing the signed distances at each grid point in the computational domain. We speed up this calculation by using a coarse sampling of the boundary for computing signed distances of grid nodes far from the boundary. For closer grid nodes, we use a finer sampling. The advantage of keeping it a distance function is that it increases the accuracy of computing spatial derivatives of $\phi$. In addition, it ensures $|\nabla \phi| \approx 1$, which increases the accuracy of computing curvature with (21) because the denominator is close to unity. Other methods exist for maintaining the distance function character of the level set (see [31], [47], [48]), but we decided to use a more straightforward approach.

The other main problem with the level set method is that, even if it is updated with a divergence free velocity field, it does not preserve mass [31]. In general, it tends to lose mass as the simulation progresses. This is mainly due to inherent numerical diffusion in the discretization of (20). We alleviate this problem by adding an appropriate constant offset to $\phi$ at each time step. This ensures global mass conservation because the constant offset affects the size of the zero level set (see Fig. 14). The mass is measured by computing the enclosed area of the zero level set, which is directly proportional to the mass (by incompressibility). If there is more than one droplet, say after a split, then different constants are added to the regions of the level set corresponding to those droplets. Hence, mass is conserved individually for each droplet. 


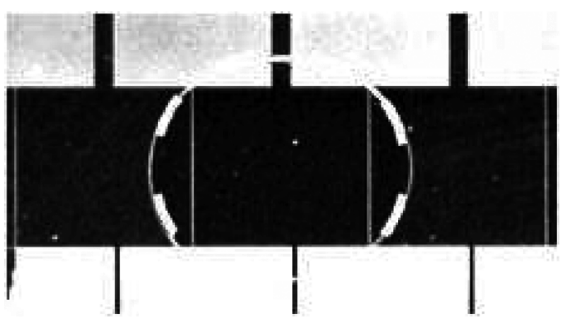

(a)

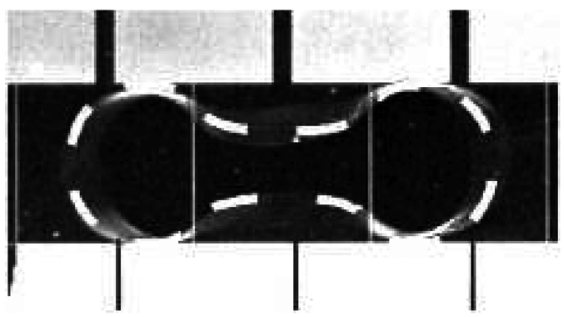

(c)

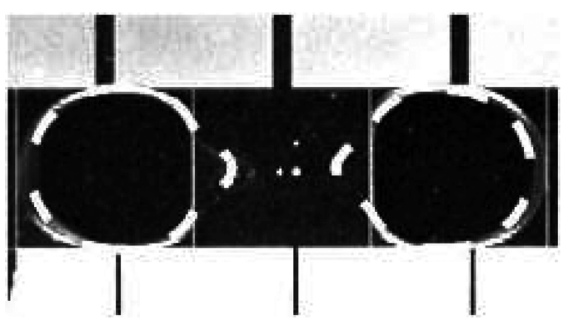

(e)

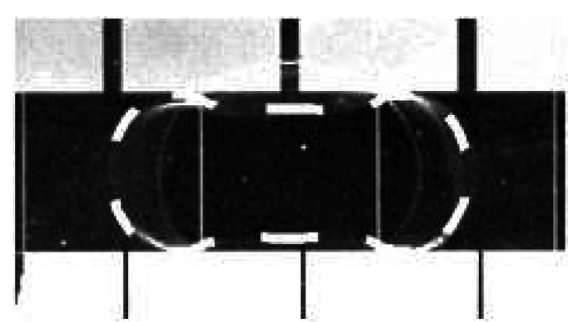

(b)

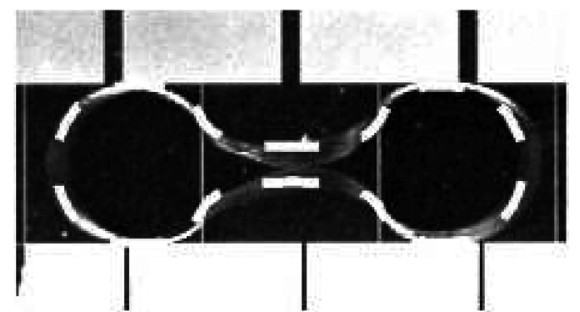

(d)

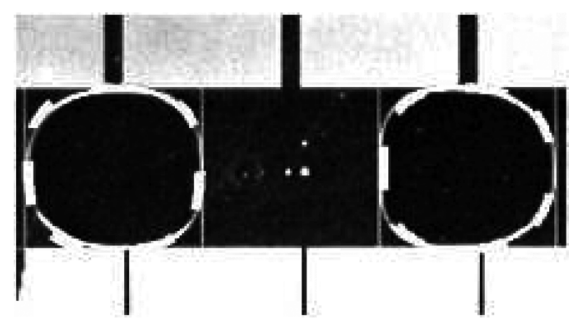

(f)

Fig. 16. Droplet splitting experimental results with simulation overlay. Six sub-frames showing the video snapshots of the experiment (courtesy of CJ Kim at UCLA). The three electrodes shown in each frame have activation voltages (from left to right) of 25,0 , and $25 \mathrm{~V}$. Each electrode is approximately square with a side length of $1.4 \mathrm{~mm}$. The dashed-line droplet outlines are from Fig. 19 and show a direct comparison between experiment and the simulation including contact angle saturation and hysteresis. (a) Time: 0 ms. (b) Time: 33.33 ms. (c) Time: 66.67 ms. (d) Time: 100 ms. (e) Time: 133.33 ms. (f) Time: 166.67 ms.

Unfortunately, the constant offset does not ensure local mass conservation, such as in the pinching region of a splitting droplet, which can cause the droplet in our simulation to 'hesitate' while splitting and sometimes get stuck. The two left and right ends would bulge and pull apart as usual, but as the neck joining them became thin it stopped moving. This was completely erroneous because the velocity field inside the droplet dictated that it should split apart. One reason for this is that the level set method does not lose mass at equal rates in different regions of the domain. Hence, a constant offset cannot properly correct for this. In addition to this, the dynamics of droplet pinching is not resolved very well because the grid resolution is fixed, uniform, and quite coarse in the pinching region (i.e., only two to five grid points).

Recently, [53] introduced a particle level set method that ensures global and local mass conservation. And in [54], a method for adaptive refinement is described that can resolve fine-scale dynamics. However, the first method is computationally intensive because of the number of seed particles needed to adequately reconstruct the level set as well as the particle reseeding routines necessary to make the algorithm work. And the second method leads to more involved data structures and coding. Therefore, we opted for the following simpler, faster method for correcting the splitting problem.

First, we check for potential splitting of the droplet by looking for thin necking regions in the flow. This is done by using infor-
TABLE III

SIMULATION PARAMETERS

\begin{tabular}{c|ccc|c} 
& $Y-L$ & Sat & All & Units \\
\hline$L$ & 4.406 & 4.406 & 4.406 & $\mathrm{~mm}$ \\
\hline$U_{0}$ & 1500 & 500 & 50 & $\mathrm{~mm} / \mathrm{sec}$ \\
\hline$t_{0}$ & 2.94 & 8.81 & 88.12 & $\mathrm{msec}$ \\
\hline$P_{0}$ & 16.3 & 16.3 & 16.3 & $\mathrm{~N} / \mathrm{m}^{2}$ \\
\hline $\mathrm{Re}$ & 117.6 & 39.2 & 3.92 & non-dim. \\
\hline $\mathrm{Ca}$ & 0.01854 & 0.006181 & 0.000618 & non-dim. \\
\hline$\tau$ & 7402.8 & 2467.6 & 246.8 & non-dim. \\
\hline$\beta$ & 47539.1 & 47539.1 & 47539.1 & non-dim.
\end{tabular}

Numbers for three simulations are listed here: (Y-L) uses the Young-Lippmann theory, (Sat) adds in saturation, and (All) includes saturation and hysteresis. Each simulation uses a different value of $U_{0}$ so that the maximum nondimensional velocity is close to unity. This also causes Re, $\mathrm{Ca}, \tau$, and $t_{0}$ to differ as well.

mation contained in the level set function, $\phi$, itself. If it is not close to splitting, then we do nothing. Otherwise, we modify $\phi$ by slightly decreasing its height in a small region around the pinch point at each time step. This prevents the level set from getting stuck and allows it to complete pinch-off without drastic modification to the level set function. In Table II, we present simulation results for the grid resolution versus time to pinch-off for the splitting case discussed in Section V-A. As can be seen the splitting time of the simulated droplet only varies by a few percent from the $108 \times 108$ grid resolution case used in Section V. 


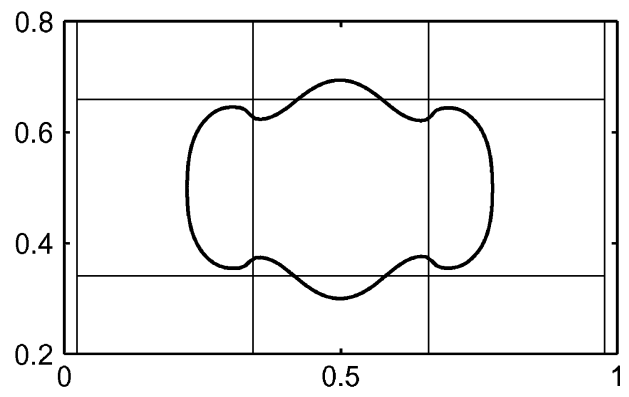

(a)

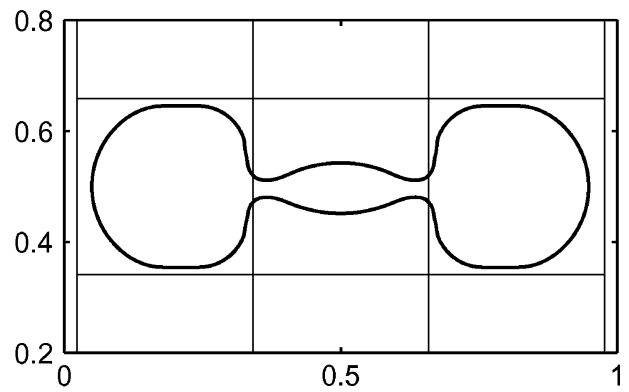

(b)

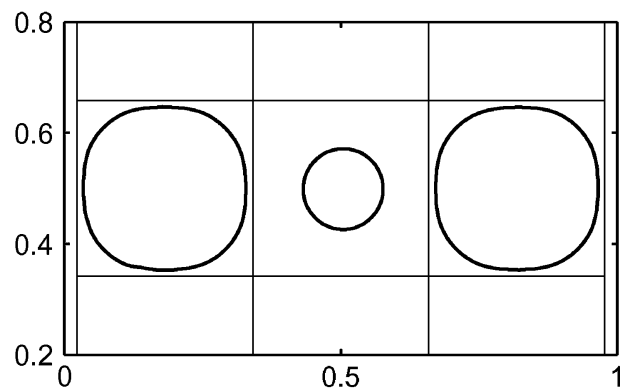

(c)

Fig. 17. Young-Lippmann model. Three simulation frames showing splitting behavior under the ideal Young-Lippmann theory: (a) time: $0.98 \mathrm{~ms}$, (b) time: $5.32 \mathrm{~ms}$, and (c) time: $10.73 \mathrm{~ms}$.

2) Choose Time Step: The next time step is chosen adaptively by the following formula [31]

$$
\Delta t=\frac{h}{\max (|u|+|v|)}
$$

where $h$ is the grid spacing of the computational domain, $u$ and $v$ are the velocities at the current time step, and the maximum is taken over all grid nodes in a thin band around the zero level set. It is not necessary to consider the whole domain because we only update level set values inside the thin band. This formula is based on the Courant-Friedrichs-Lewy (CFL) condition, which specifies the largest time step that can be taken and still allow the simulation to remain stable [55]. It is common to have some safety margin by choosing a smaller time step, but this is unnecessary because the HJ-WENO method in Section IV-C1 has built-in artificial dissipation which enhances stability. For more details, see [31] and [55].

\section{RESULTS: THEORY VERSUS EXPERIMENT}

In the following sections, the physics of the splitting droplet experiment are described in detail. Next, modeling and simu-

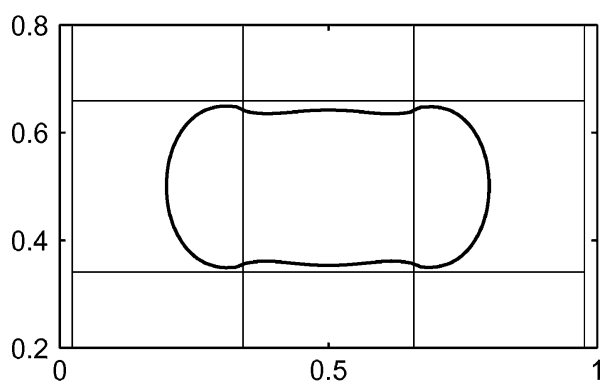

(a)

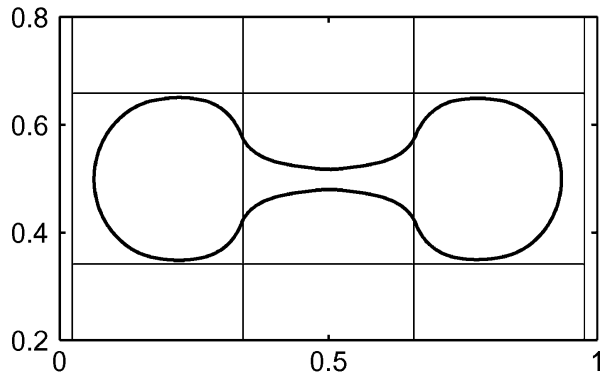

(b)

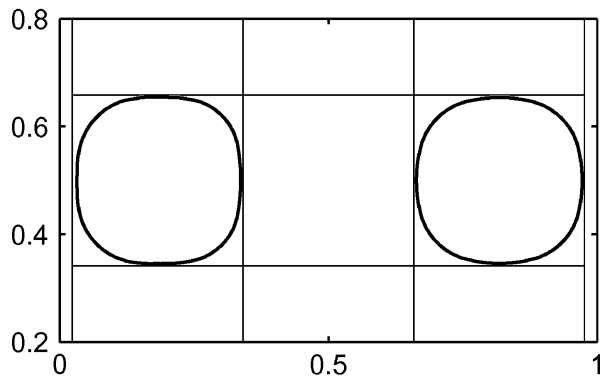

(c)

Fig. 18. Saturation model. Three frames showing simulation results when saturation is included: (a) time: $2.72 \mathrm{~ms}$, (b) time: $10.68 \mathrm{~ms}$, and (c) time: 17.47 ms.

lation results of the experiment are presented. We discuss the various physical phenomena affecting the motion of the droplet (i.e., saturation and hysteresis) and how the simulations were modified to capture these effects. Finally, we compare our simulation with a different EWOD experiment to show that a model developed for one type of experiment is predictive for a new experiment.

\section{A. Theory, Simulation, and Experiment for a Splitting Droplet}

In Fig. 16, an overhead view of an EWOD device with three electrodes running left to right is depicted with a splitting droplet. The voltage actuation, from left to right, is 25,0 , and $25 \mathrm{~V}$ and is constant throughout the split. In frame (a), an initial near circular droplet is shown just before voltage activation. After the voltage is turned on, the liquid-gas interface over the left and right electrodes deforms and induces a low pressure region there. The regions where no voltage is activated remain at high pressure. In the subsequent frames, the droplet is pulled from the left and right sides, while it is pushed in from the top and bottom. The droplet elongates along the horizontal dimension and is being pinched in the vertical direction. This causes two smaller droplets to form on the left and right sides, with a 


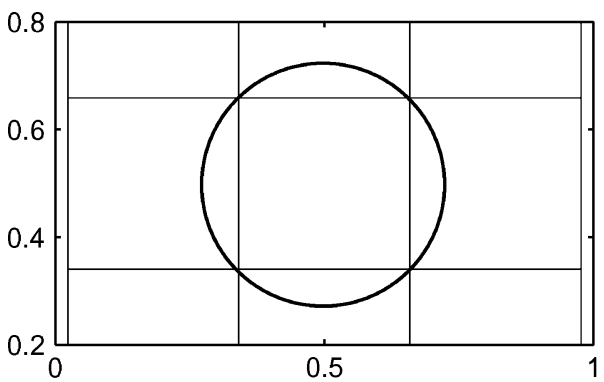

(a)

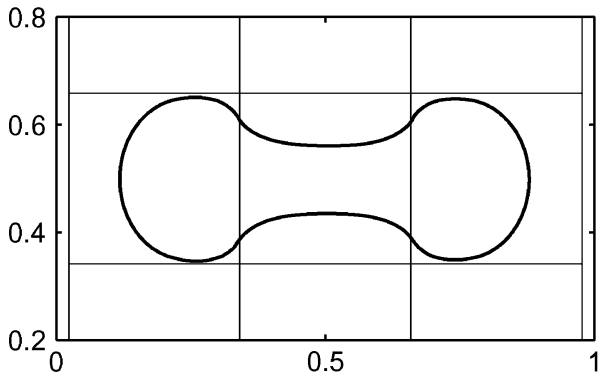

(c)

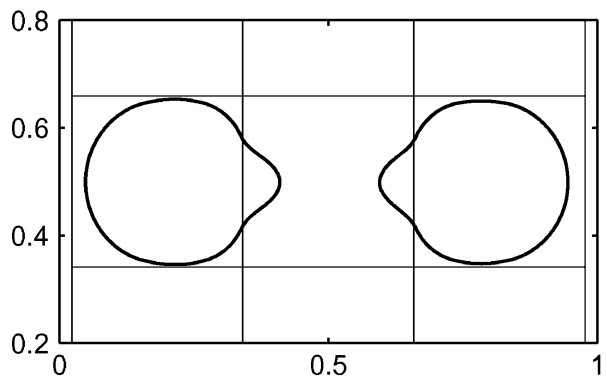

(e)

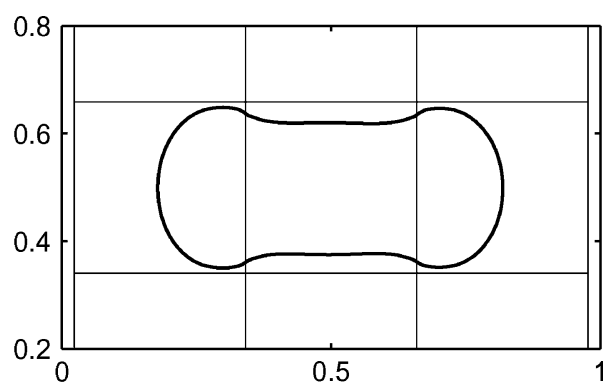

(b)

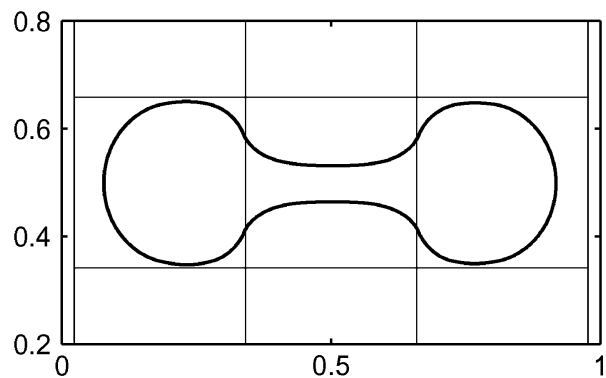

(d)

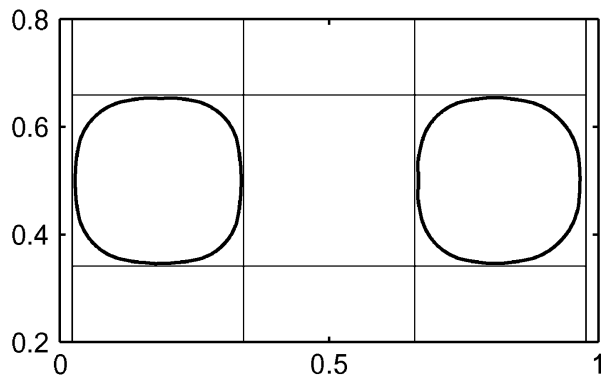

(f)

Fig. 19. Simulation results. Six subframes showing simulation snapshots when saturation and hysteresis are included (each frame corresponds to the experimental frames in Fig. 16): (a) time: $0.00 \mathrm{~ms}$, (b) time: $33.33 \mathrm{~ms}$, (c) time: $66.67 \mathrm{~ms}$, (d) time: $100.00 \mathrm{~ms}$, (e) time: $133.33 \mathrm{~ms}$, and (f) time: $166.67 \mathrm{~ms}$.

thin neck joining them. The neck eventually gets so thin that it snaps due to capillary instability. The two smaller droplets then continue moving to the left and right electrodes because of the pressure differential created from the voltage actuation. Finally, the two droplets come to rest on the two $25 \mathrm{~V}$ electrodes. The total time of this experiment is approximately $167 \mathrm{~ms}$.

Next, we present three simulations showing the effects of the various physics at the boundary. The first simulation is for contact angle variations obeying the ideal Young-Lippmann curve (see the Y-L curve in Fig. 9). Next, we simulate droplet splitting assuming only contact angle saturation (see the saturation curve for two plates in Fig. 9). Finally, we show a simulation that includes saturation and hysteresis. In Table III, we list the pertinent parameters of each simulation. The velocity scale, $U_{0}$, is chosen so that the maximum nondimensional velocity during the simulation is close to unity.

1) Ideal Young-Lippmann: In Fig. 17, we have a simulation of droplet motion when no contact angle saturation or hysteresis is being modeled. As can be seen, the general shape of the splitting droplet is not the same as in the experiment. In frame (a), just after the voltage is turned on, the droplet shape has much more of a bulge in the center than shown in the experiment. This becomes more pronounced in frame (b), with two thin necks de- veloping between the three bulging parts of the droplet. Finally, in frame (c), the droplet has split into three pieces instead of two as in the experiment. Also, the total time to complete the split and reach equilibrium is $10.73 \mathrm{~ms}$, which is 15.6 times faster than the experiment.

Since no saturation or hysteresis is being modeled, the simulated EWOD force is much larger than it is in reality. This causes the droplet to be pulled apart so fast, that the middle region is never able to become a thin neck. As a result, three satellite droplets are created instead of two. In fact, the $z$ curvature of the liquid-gas interface (i.e., the EWOD force) is so large that the $x$ - $y$ curvature component is practically negligible. This is why the droplet does not resist being pinched in two places; the EWOD force here dominates the large curvature forces induced by the pinched regions.

2) Saturation: For the simulation shown in Fig. 18, we have added the effect of contact angle saturation. The splitting motion of the droplet now looks much closer to the experiment. As the droplet is pulled apart, a single thin neck joins the two bulging ends. The neck then breaks, allowing the two droplets to come to rest on the left and right electrodes. However, the time scale is still not correct. The time to reach equilibrium here is 17.47 ms, which is 9.6 times faster than the experiment. 


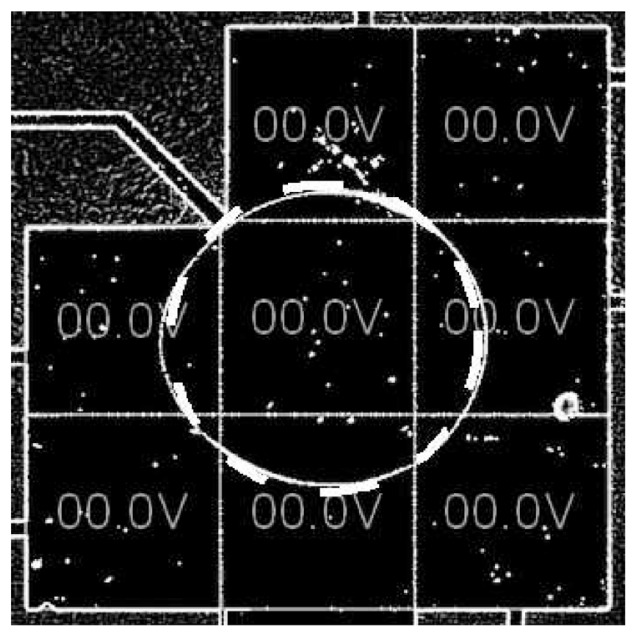

(a)

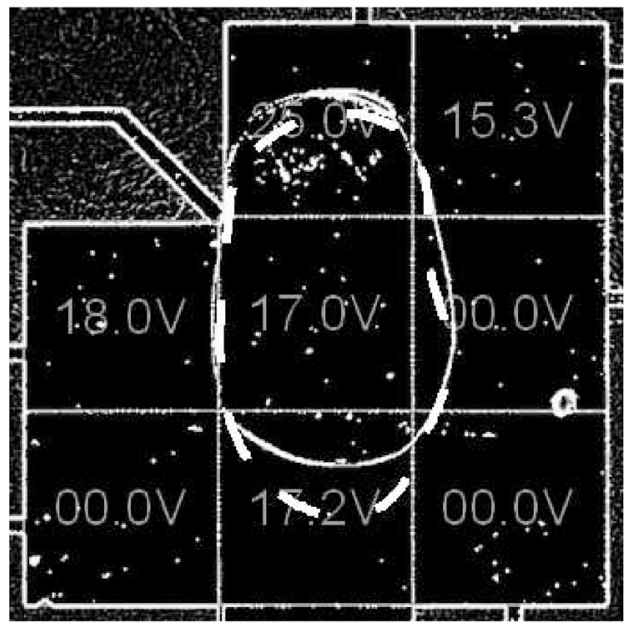

(c)

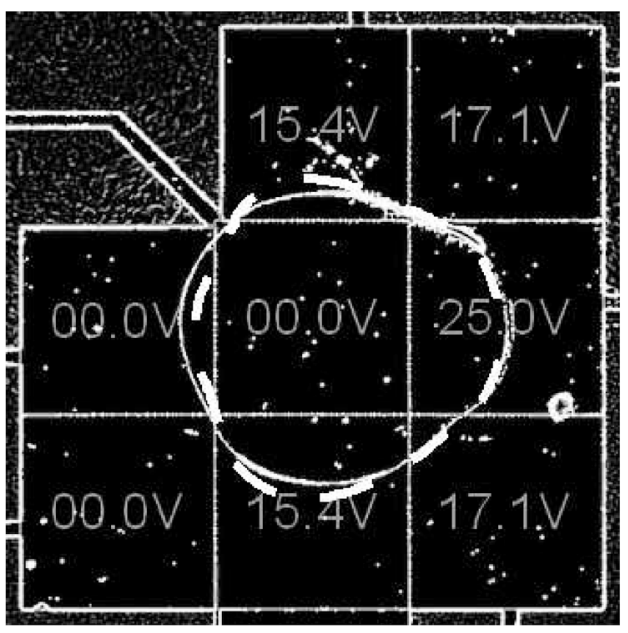

(b)

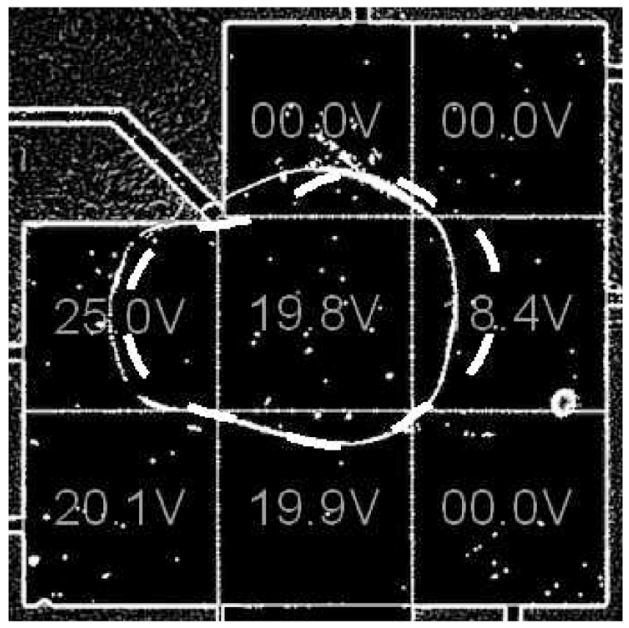

(d)

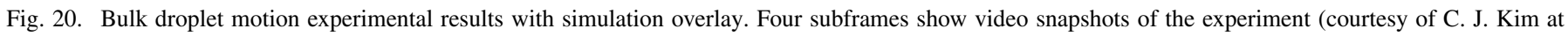

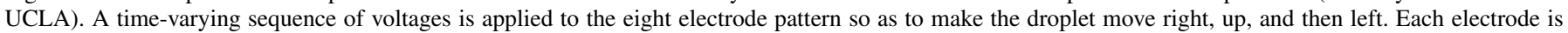

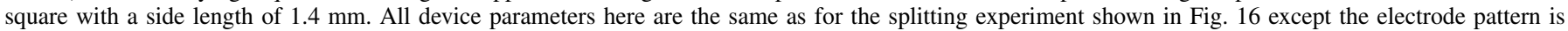

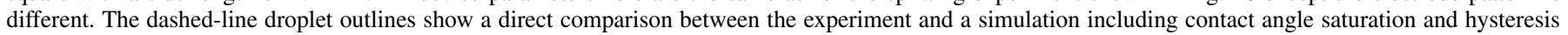
$\left(K_{\mathrm{hys}}=0.09\right)$. (a) Time: $0.0 \mathrm{~ms}$. (b) Time: $31.6 \mathrm{~ms}$. (c) Time: $79.9 \mathrm{~ms}$. (d) Time: $136.6 \mathrm{~ms}$.

Including saturation does slow the droplet down, but it is still not enough. In reality, the line pinning induced contact angle hysteresis is extremely significant in all wetting phenomena. Hysteresis slows down motion by reducing the pressure gradient (see Section III-B4). Therefore, it is not surprising that this effect must be included to accurately simulate droplet speed. The following section presents our final simulation of splitting with hysteresis included.

3) Contact Angle Hysteresis: In Fig. 19, we show six snapshots of our simulated splitting droplet, which are also shown as dashed-line overlays in Fig. 16. The hysteresis constant, $K_{\text {hys }}$, is 0.09. This simulation is similar to the one in Fig. 18, except that the time scale is now correct. The simulated droplet now splits in the same amount of time as the experiment, as shown in Fig. 16.

The value of the hysteresis constant, $K_{\text {hys }}=0.09$, was chosen to make the simulation time scale match the experiment. By using (12) and experimental data from Fig. 9, we estimate the contact angle deflection due to hysteresis to be
$\Delta_{\text {hys }}=6.4^{\circ}$. In [56], they give a value of about $20^{\circ}$ for sessile drops of water sliding on top of a Teflon surface. This discrepancy is reasonable since the droplet size and geometry in the EWOD device is different than in [56].

Our hysteresis constant is also analogous to the contact line friction coefficient in [28], where they treat contact line friction as an extra forcing term that is proportional to the velocity of the contact line. In their case, the forcing term has units of force per unit contact line length. By scaling their friction force by the ratio of contact line length to volume for a droplet in an EWOD device (to put it into units of force per unit volume), we can include this as a body force term in the Navier-Stokes equations. After going through the same derivation in Section III-A2 we obtain equations similar to (6) and (7), except the coefficient of the velocity term has an extra positive term added to $12(L / H)^{2}$. Hence, the coefficient is larger than before. If we ignore the velocity time derivative term in (6) and (7), the extra friction force is equivalent to multiplying the pressure gradient by a constant smaller than one (i.e. $K_{\text {hys }}$ ). In fact, one can show 
that our choice of hysteresis constant corresponds to a contact line friction coefficient of 0.5664 Newton-seconds per square meter, which is comparable to the values listed in [28] for a column of fluid comprised of deionized water between two parylene coated electrodes.

However, one must keep in mind that these estimates are rough approximations. The hysteresis constant is not an exact model nor does it capture line pinning. But our goal was not to model line pinning or contact angle hysteresis in great detail. The point is that a simple scaling constant is all that is needed to produce simulations that approximately capture the shape and speed of droplet motion.

\section{B. Simulation Versus Moving Droplet Experiment}

In this section, we compare our simulation to another experimental case to give more supporting evidence for our model. The EWOD device shown here has eight electrodes arranged in a square-like pattern. A predetermined voltage sequence was used to actuate the droplet so that it moves to the right first, then up, and finally to the left. All constants in our simulation are the same as those used in Section V-A3 (i.e., $K_{\text {hys }}=0.09$ ) and the same computational grid resolution $(108 \times 108)$ is used.

From Fig. 20, it is evident that the simulation follows the experiment fairly well. The match is not exact, however, and this is mainly because line pinning is not taken into account in our model. But the overall motion and time scale is correct.

\section{CONCLUSION}

In this paper, we have presented a model and numerical simulation of droplet motion inside an electrowetting device. Starting from the full Navier-Stokes equations we obtained a reduced order model, similar to Hele-Shaw type flow, that captures the bulk dynamic behavior of EWOD driven droplets in two dimensions. The key part of our analysis is including contact angle saturation and a simple, computationally efficient model of hysteresis in order to match the experimental data. Our simulation results show how these two physical phenomena affect the motion of the droplet. When all effects are included, our simulations compare favorably with the experiments, but are not an exact match. The main reason for this is our model does not include contact line pinning, which is observable in the experiments. Our numerical implementation is fast, simple, and readily lends itself to control algorithm design. By using the level set method, our simulation is able to easily capture droplet splitting. The computing times of all simulations (in MATLAB) varied between three and six minutes, which shows the speed of our method.

\section{ACKNOWLEDGMENT}

The authors would like to thank C.-J. Kim, R. Garrell, S. $\mathrm{K}$. Cho, and J. Gong for providing us with information on the EWOD device and experimental data for splitting and moving droplets.

\section{REFERENCES}

[1] B. He and J. Lee, "Dynamic wettability switching by surface roughness effect," in Proc. 16th Annu. IEEE Int. Conf. MEMS, Kyoto, Japan, Jan. 2003, pp. 120-123.
[2] A. A. Darhuber, J. M. Davis, S. M. Troian, and W. W. Reisner, "Thermocapillary actuation of liquid flow on chemically patterned surfaces," Phys. Fluids, vol. 15, no. 5, pp. 10150-10153, 2003.

[3] N. Fortner, B. Shapiro, and A. Hightower, "Modeling and passive control of channel filling for micro-fluidic networks with thousands of channels," in American Institute of Aeronautics and Astronautics (AIAA) 33rd AIAA Fluid Dynamics Conference and Exhibit, Orlando, FL, June 23-26, 2003.

[4] G. Lippmann, "Relation entre les phenomenes electriques et capillaires," Ann. Chim. Phys., vol. 5, pp. 494-549, 1875

[5] F. Mugele and J.-C. Baret, "Electrowetting: From basics to applications," J. Phys.: Condensed Matter, vol. 17, pp. R705-R774, 2005.

[6] A. A. Darhuber and S. M. Troian, "Principles of microfluidic actuation by modulation of surface stresses," Annu. Rev. Fluid Mech., vol. 37, pp. $425-455,2005$.

[7] A. Klingner and F. Mugele, "Electrowetting-induced morphological transistions of fluid microstructures," J. Appl. Phys., vol. 95, no. 5, pp. 2918-2920, 2004.

[8] A. Klingner, J. Buehrle, and F. Mugele, "Capillary bridges in electric fields," Langmuir, vol. 20, pp. 6770-6777, 2004

[9] W. Satoh, M. Loughran, and H. Suzuki, "Microfluidic transport based on direct electrowetting," J. Appl. Phys., vol. 96, no. 1, pp. 835-841, 2004.

[10] V. K. Pamula, P. Paik, J. Venkatraman, M. G. Pollack, and R. B. Fair, "Microfluidic electrowetting-based droplet mixing," in Proc. Micro Electro Mech. Syst. Conf., Berkeley, CA, 2001.

[11] H. Ren and R. B. Fair, "Micro/nano liter droplet formation and dispensing by capacitance metering and electrowetting actuation," Proc. IEEE-NANO Tech. Dig. , pp. 369-372, 2002

[12] R. B. Fair, V. Srinivasan, H. Ren, P. Paik, V. K. Pamula, and M. G. Pollack, "Electrowetting-based on-chip sample processing for integrated microfluidics," Proc. IEEE Int. Electron Devices Meeting (IEDM), 2003.

[13] V. Srinivasan, V. K. Pamula, M. G. Pollack, and R. B. Fair, "A digital microfluidic biosensor for multianalyte detection," in Proc. IEEE 16th Annu. Int. Conf. Micro Electro Mech. Syst., 2003, pp. 327-330.

[14] F. Cattaneo, K. Baldwin, S. Yang, T. Krupenkine, S. Ramachandran, and J. A. Rogers, "Digitally tunable microfluidic optical fiber devices," J. Microelectromech. Syst., vol. 12, no. 6, pp. 907-912, 2003.

[15] J. Hsieh, P. Mach, F. Cattaneo, S. Yang, T. Krupenkine, K. Baldwin, and J. A. Rogers, "Tunable microfluidic optical-fiber devices based on electrowetting pumps and plastic microchannels," IEEE Photon. Technol. Lett., vol. 15, no. 1, pp. 81-83, 2003.

[16] T. Roques-Carmes, R. A. Hayes, B. J. Feenstra, and L. J. M. Schlangen, "Liquid behavior inside a reflective display pixel based on electrowetting," J. Appl. Phys., vol. 95, no. 8, pp. 4389-4396, 2004.

[17] S. K. Cho, H. Moon, and C.-J. Kim, "Creating, transporting, cutting, and merging liquid droplets by electrowetting-based actuation for digital microfluidic circuits," J. Microelectromech. Syst., vol. 12, no. 1, pp. 70-80, 2003.

[18] L. Minnema, H. A. Barneveld, and P. D. Rinkel, "An investigation into the mechanism of water treeing in polyethylene high voltage cables," IEEE Trans. Electr. Insul., vol. EI-15, pp. 461-472, 1980

[19] B. Berge, "Electrocapillarité et mouillage de films isolants par l'eau,' Comptes Rendus de l'Académie des Sciences de Paris, , ser. II, vol. 317, pp. 157-163, 1993, English translation available.

[20] A. R. Wheeler, H. Moon, C.-J. Kim, J. A. Loo, and R. L. Garrell, "Electrowetting-based microfluidics for analysis of peptides and proteins by matrix-assisted laser desorption/ionization mass spectrometry," Anal. Chem., vol. 76, pp. 4833-4838, 2004.

[21] J. Fowler, H. Moon, and C. J. Kim, "Enhancement of mixing by droplet-based microfluidics," in Proc. IEEE Conf. MEMS, 2002, pp. 97-100.

[22] J. Gong, S. K. Fan, and C. J. Kim, "Portable digital microfluidics platform with active but disposable lab-on-chip," in Proc. IEEE Conf. MEMS, Maastricht, The Netherlands, Jan. 2004, pp. 355-358.

[23] K. Hoshino, S. Triteyaprasert, K. Matsumoto, and I. Shimoyama, "Electrowetting-based actuation for microinjection," in 12th Int. Conf. Solid-State Sens., Actuators Microsyst. (Transducers), Boston, MA, Jun. 8-12, 2003, pp. 1800-1803.

[24] S. K. Cho and C. J. Kim, "Particle separation and concentration control for digital microfluidic systems," in Proc. IEEE 16th Annu. Int. Conf. MEMS, Kyoto, Japan, Jan. 2003, pp. 686-689.

[25] H. J. J. Verheijen and W. J. Prins, "Reversible electrowetting and trapping of charge: Model and experiments," Langmuir, vol. 15, pp. 6616-6620, 1999. 
[26] B. Shapiro, H. Moon, R. Garrell, and C. J. Kim, "Equilibrium behavior of sessile drops under surface tension, applied external fields, and material variations," J. Appl. Phys., vol. 93, 2003.

[27] T. B. Jones, "On the relationship of dielectrophoresis and electrowetting," Langmuir, vol. 18, pp. 4437-4443, 2002.

[28] K.-L. Wang and T. B. Jones, "Electrowetting dynamics of microfluidic actuation," Langmuir, vol. 21, no. 9, pp. 4211-4217, 2005.

[29] C. Decamps and J. D. Coninck, "Dynamics of spontaneous spreading under electrowetting conditions," Langmuir, vol. 16, no. 26, pp. 10150-10153, 2000.

[30] H.-W. Lu, K. Glasner, A. L. Bertozzi, and C.-J. Kim, "A diffuse interface model for electrowetting droplets in a hele-shaw cell," J. Fluid Mech., 2005, submitted for publication.

[31] S. Osher and R. Fedkiw, Level Set Methods and Dynamic Implicit Surfaces. New York, NY: Springer-Verlag, 2003.

[32] G. E. Karniadakis and A. Beskok, Micro Flows: Fundamentals and Simulation. New York, NY: Springer-Verlag, 2001.

[33] R. L. Panton, Incompressible Flow, 2nd ed. New York, NY: Wiley, 1996.

[34] G. K. Batchelor, An Introduction to Fluid Dynamics. New York, NY: Cambridge University Press, 1967.

[35] M. P. do Carmo, Differential Geometry of Curves and Surfaces. Upper Saddle River, NJ: Prentice Hall, 1976.

[36] N. S. Nise, Control Systems Engineering, 2nd ed. Redwood City, CA: The Benjamin/Cummings Publishing Company, Inc., 1995.

[37] R. S. Burdon, Surface Tension and the Spreading of Liquids. Cambridge, U.K.: Cambridge University Press, 1949.

[38] E. G. Pétré and A. Sanfeld, Lecture Notes in Physics: Capillarity Today. New York, NY: Springer-Verlag, 1990.

[39] E. J. Drelich, J. S. Laskowski, and K. L. Mittal, Apparent and Microscopic Contact Angles. Zeist, The Netherlands: VSP BV, 2000.

[40] P.-G. de Gennes, F. Brochard-Wyart, and D. Quéré, Capillarity and Wetting Phenomena: Drops, Bubbles, Pearls, Waves. New York, NY: Springer-Verlag, 2004.

[41] C. J. Kim and J. Lee, "Microactuation by continuous electrowetting phenomenon and silicon deep rie process," in Proc. ASME Inte. Mech. Eng. Congr. Expo., Anaheim, CA, 1998.

[42] — - "Liquid micromotor driven by continuous electrowetting," in Proc. IEEE Micro Electro Mechanical Syst. Workshop, Heidelberg, Germany, 1998.

[43] M. Vallet, B. Berge, and L. Vovelle, "Electrowetting of water and aqueous solutions on poly(ethyleneterephthalate) insulating films," European Phys. J., vol. 11, pp. 583-591, 1996.

[44] E. Seyrat and R. A. Hayes, "Amorphous fluoropolymers as insulators for reversible low-voltage electrowetting," J. Appl. Phys., vol. 90, 2001.

[45] T. D. Blake, A. Clarke, and E. H. Stattersfield, "An investigation of electrostatic assist in dynamic wetting," Langmuir, vol. 16, no. 6, pp. $2928-2935,2000$.

[46] J. M. Floryan and H. Rasmussen, "Numerical methods for viscous flows with moving boundaries," Appl. Mech. Rev., vol. 42, no. 12, pp. 323-340, 1989.

[47] S. A. Sethian, Level Set Methods and Fast Marching Methods, 2nd ed. New York, NY: Cambridge University Press, 1999.

[48] R. Caiden, R. Fedkiw, and C. Anderson, "Numerical method for two phase flow consisting of separate compressible and incompressible regions," J. Comput. Phys., vol. 166, pp. 1-27, 2001.
[49] R. P. Fedkiw, T. Aslam, B. Merriman, and S. Osher, "A non-oscillatory eulerian approach to interfaces in multi-material flows (the ghost fluid method)," J. Comput. Phys., vol. 152, pp. 457-492, 1999.

[50] A. Ralston and P. Rabinowitz, A First Course in Numerical Analysis, 2nd ed. Mineola, NY: Dover, 2001.

[51] G. Evans, J. Blackledge, and P. Yardley, Numerical Methods for Partial Differential Equations. London, U.K.: Springer-Verlag, 2000.

[52] G. D. Smith, Numerical Solution of Partial Differential Equations, Third ed. Oxford, U.K.: Oxford University Press, 1985.

[53] D. Enright, R. P. Fedkiw, J. Ferziger, and I. Mitchell, "A hybrid particle level set method for improved interface capturing," J. Comput. Phys., vol. 183, pp. 83-116, 2002.

[54] F. Losasso, F. Gibou, and R. Fedkiw, "Simulating water and smoke with an octree data structure," in ACM Trans. Graph. (SIGGRAPH Proc.), Los Angeles, CA, 2004.

[55] K. W. Morton and D. F. Mayers, Numerical Solution of Partial Differential Equations. New York, NY: Cambridge University Press, 1994.

[56] E. J. F. Padday, Wetting, Spreading, and Adhesion. London: Academic, 1978

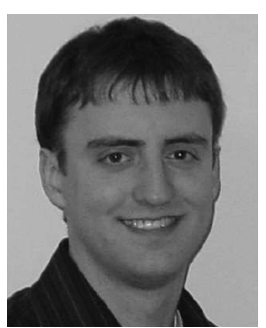

Shawn W. Walker received the B.S. degree in electrical engineering from Virginia Polytechnic Institute and State University (Virginia Tech), Blacksburg, in 1998 and the M.S. degree in engineering and applied science from Yale University, New Haven, CT, in 2002. He also worked at Raytheon Electronic Systems from 1998 to 2000 as a hardware design and antenna control systems engineer. Currently, he is completing the Ph.D. degree in the Department of Aerospace Engineering at the University of Maryland (UMD), College Park.

His main research interests are in applied mathematics, numerics, systems modeling, and control.

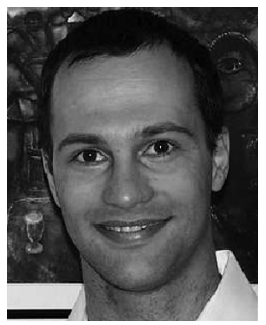

Benjamin Shapiro (M'04) was born in Jerusalem, Israel, in 1973. He received the B.S. degree in aerospace engineering from the Georgia Institute of Technology, Atlanta, in 1995, and the Ph.D. degree in control and dynamical systems from the California Institute of Technology, Pasadena, in 1999.

He joined the Department of Aerospace Engineering, University of Maryland, College Park, as an Assistant Professor in 2000 and is now an Associate Professor. His main interests are in modeling and control of microsystems, with a primary focus on modeling and control of microfluidic systems for biochemical applications. He is part of the interdisciplinary Small Smart Systems Center and is affiliated with the Bioengineering Graduate Program and the Applied Math And Scientific Computation Program at the University.

Dr. Shapiro was the organizer of the March 2004 NSF workshop on Control and System Integration of Micro- and Nano-Scale Systems and is a recipient of a 2003 NSF CAREER Award. 